

\section{Debate, confrontación y hegemonía en la disciplina de la comunicación. Nuevos tiempos para un paradigma dominante}

Debate, confrontation and hegemony in the Communication field. New era for a dominant paradigm in media research DOI: 10.15213/redes.n1o.p145

ÁNGEL CARRASCO CAMPOS, ENRIC SAPERAS

\section{ABSTRACT}

In many ways communication research reflects the debates, confrontations and times of hegemony that have shaken the social sciences in its institutionalization process during the last century. Looking back to the immediate past of media studies allows us to observe the evolution of contemporary social theory, while the current changes imposed by globalization reposition in the central debate the theoretical and institutional transformations of international communication research. Its multidisciplinary structure, organizational capacity, proximity to knowledge needs applied to the ever expanding industrial and cultural sectors, the centrality of its objects of study and its predictive potential were only possible by means of a socially agile behaviour, a great capacity for renewal, and a permanent discussion about its disciplinary field. Therefore, the use of theory in communication research occupies a central position in its ability to explain, interpret and understand communication processes. The disputes between the organization of instrumental theories and the development of comprehensive, critical or emancipatory theories have always focused the interest of communication researchers with a common goal: achieving dominance in the discipline. In this paper we analyse the evolution of the so called "dominant paradigm" in three moments of dispute with critical and interpretative theories: the period started with the interventionist and predictive social policies of the New Deal, its renewal process against the balkanization of media research during the sixties, and the formulation of a rejuvenated dominant paradigm in the present times of globalization. We will focus on this last period of new 
institutionalization and standardization of international communication research.

KEYWORDS: COMMUNICATION THEORY, COMMUNICATION RESEARCH, DOMINANT PARADIGM, INSTITUTIONALIZED RESEARCH, GLOBALIZATION

\section{RESUMEN}

La investigación comunicativa refleja en muchos aspectos los debates, confrontaciones y tiempos de hegemonía que han sacudido las ciencias sociales en su proceso de institucionalización en el último siglo. Una mirada hacia el pasado inmediato de los estudios sobre medios nos permite observar los rasgos de evolución de la teoría social contemporánea, al tiempo que las actuales transformaciones impuestas por la globalización vuelvena situar el devenir teórico e institucional de la investigación comunicativa internacional en el centro del debate. Su estructura pluridisciplinar, su capacidad organizativa, la proximidad a necesidades de conocimiento aplicado en un sector industrial y cultural siempre en expansión, la centralidad de sus objetos de estudio y su potencialidad predictiva sólo han sido posibles por cuanto se ha comportado como una disciplina socialmente ágil, con gran capacidad de renovación,y dotada de habilidad en la discusión de su campo disciplinar. Por ello, el uso de la teoría en la investigación comunicativa ocupa una posición central en su capacidad de explicar, interpretar y comprender los procesos de comunicación. Las disputas por organizar teorías instrumentales y aplicadas, o por elaborar teorías comprensivas, críticas o emancipatorias siempre han centrado el interés de los investigadores de la comunicación con un mismo objetivo: conseguir el dominio en la disciplina. En este texto analizamos la evolución de lo que se ha dado en denominar "paradigma dominante" en sus tres periodos de disputa con las teorías críticas e interpretativas: el periodo iniciado con las políticas intervencionistas y predictivas en lo social del New Deal, en su proceso renovación frente a las tendencias de balcanización de la investigación en los años sesenta, y la formulación de un remozado paradigma dominante en los actuales tiemposde globalización. Centraremos nuestra atención en este último periodo de nueva institucionalización y estandarización internacional de la investigación comunicativa.

PALABRAS CLAVE: TEORÍA DE LA COMUNICACIÓN, INVESTIGACIÓN COMUNICATIVA, PARADIGMA DOMINANTE, INVESTIGACIÓN INSTITUCIONALIZADA, GLOBALIZACIÓN 


\section{LOS USOS DE LA TEORía EN LA INVESTIGACión de LA COMUNiCACIÓn: GÉNESIS Y CONSOLIDACIÓN DEL PARAdIGMA DOMINANTE}

Desde sus orígenes, la cienciase ha distinguido de otras formas de conocimiento por la aplicación de un método concreto (el método científico) en su mirada hacia la realidad. Mientras, los diferentes ámbitos científicos han empleado un doble criterio de demarcación: por una parte, el propio objeto de estudio (pudiendo distinguir diferentes ciencias con base en una determinada y contingente parcelación del todo de la realidad sobre la cual focalizar la atención); por otra parte, la propia construcción teórica generada a partir de hipótesis y resultados en la aplicación del método científico sobre objetos concretos. Las teorías, por tanto, han tenido y tienen un carácter demarcador en el ámbito de las ciencias y es, entre otros criterios, a partir de la construcción de un corpus teórico propio como los académicos podemos identificar el reconocimiento de un ámbito científico con pretensiones de autonomía epistemológica.

En el caso de la investigación de la comunicación, caracterizada no tanto como ciencia específica sino como ámbito de investigación pluridisciplinar, su estatuto científico ha sido siempre objeto de debate, tanto por la propia realidad cambiante de su objeto de estudio (con cambios en el propio sistema comunicativo, a nivel económico, tecnológico, político y cultural), como por la propia discusión académica a la hora de determinar un cuerpo teórico común. En este debate epistemológico, las teorías y sus usos por parte de los investigadores han desempeñado un papel determinante a la hora de definir el para qué de las ciencias de la comunicación. Por tanto, la propia comprensión de la actividad investigadora y su interés del conocimiento, en el sentido de Jürgen Habermas (1986), puede ser rastreada sintomáticamente a través de las construcciones, usos y sistematizaciones de las teorías de la comunicación que los investigadores hacen como parte de la práctica investigadora en su conjunto.

La determinación del campo y del valor desempeñado por la teoría será uno de los primeros escenarios de debate durante los primeros pasos de la investigación de la comunicación, en los Estados Unidos de la década de 1930. En un contexto de salida de la Gran Depresión mediante las políticas económicas del New Deal, y en un extenso país multicultural en el que la radiodifusión en los hogares se planteaba, más allá de un medio para la información y el entretenimiento, como elemento sobre el que poder construir mecanismos de integración social,el debate acerca del papel de los investigadores de la comunicación adquiere una dimensión que rebasa lo 
puramente académico, apuntando hacia modelos de intervención en lo social. Es en este espacio en el que debemos situar el debate epistemológico, casi fundacional, entre funcionalismo y Teoría Crítica; en juego quedaba el lugar de la teoría bien como "fuerza productiva práctica, transformadora" (Adorno, 2003: 165) que "no se limita a aceptar pasivamente en cada caso lo dado", (Adorno, 2003: 177), bien como propuesta al servicio del propio objeto (en este caso, del sistema comunicativo), sus instituciones y sus intereses:

"Antes y además de servir a cualquier propósito específico, debería estudiarse el papel general de los medios de comunicación en el sistema social actual” (Lazarsfled, 1941: 9).

"La totalidad del mundo perceptible, tal como existe para el miembro de la sociedad burguesa y tal como se interpreta en la concepción tradicional del mundo que se encuentra en interacción, con aquélla, se presenta al sujeto como un conjunto de facticidades; el mundo está ahí y debe ser aprehendido. El pensar organizador de cada individuo pertenece al conjunto de las relaciones so-ciales que tienden a adaptarse del modo más adecuado posible a las necesidades. Pero entre el individuo y la sociedad existe en este punto una diferencia esencial" (Horkheimer, 2003: 34-35).

El resultado de dicho debate se saldaría con la consolidación del estructural-funcionalismo como paradigma dominante de investigación, a modo de investigación administrada inscrita en un programa decidido de cooperación (Ethic of Cooperation) entre las instituciones y la academia. La propuesta investigadora sentaba las bases de un programa epistemológico, hegemónico hasta la actualidad, caracterizado por la eficacia de su sistematización, su alta aplicabilidad y su fuerte capacidad explicativa y predictiva, y basado en la construcción teórica como interrelación de hipótesis parciales en torno a efectos observables, medibles y predecibles. La teoría quedaba definida así como un momento del proceso investigador al servicio instrumental de los intereses empíricos (orientados administradamente - Gitlin, 1978), y se postulaba como programa de investigación de integración, con voluntad de superación del debate en torno a los intereses de investigación (ya sean los emancipatorios defendidos por Teoría Crítica, o los comprensivos propuestos por el interaccionismo simbólico). 
La Mass Communication Research norteamericana incorpora así el modelo de la american science (en detrimento de las tradiciones europeas clásicas), en la que destaca la construcción teórica a partir teorías de rango intermedio (middle range theories) o teorías especiales (special theories). Este modelo será consolidado, ordenado y sistematizado con la primera edición de 1949 del clásico Social Theory and Social Structure de R.K. Merton, para el que las teorías de rango intermedio, siguiendo el modelo explicativo y predictivo de las ciencias sociales aplicadas según la caracterización de John Maynard Keynes (1931), se definen frente a los grandes sistemas conceptuales de la filosofía y la sociología primitiva (con aspiraciones de dar cuenta del todo social), como los modelos teóricos desde los cuales es posible el avance empírico de la ciencia sin caer en la especulación. El valor de la teoría se determina así como elemento subsidiario al objeto de estudio, y se propone como elemento integrador y legitimador de los diferentes modos de hacer ciencia de lo social. Desde esta perspectiva no hay cabida para paradigmas investigadores enfrentados por intereses de conocimiento, sino una gran distinción entre grandes sistemas teóricos ("total systems of sociological theory") que se demuestran estériles e inconsistentes en su aplicación práctica, y teorías de rango intermedio capaces de avances significativos al proceder en el doble plano de construcción teórica mediante hipótesis susceptibles de ser objeto de investigación empírica ("hypotheses that can be empirically investigated") y la progresiva interrelación de las diferentes teorías intermedias y parciales que superen el test falsacionista.(Merton, 1949: 39-53).

La transformación del sistema político, económico, ideológicoy mediático en la década de los años sesenta(evidenciado en el desarrollo de la Guerra Fría y los procesos de descolonización, la transformación del audiovisual, el cine en gran pantalla y la radiofórmula musical) situará a la investigación comunicativa ante su primera gran crisis teórica, por cuanto el principal modelo dominante hasta la fecha (investigación de efectos bajo el modelo de Personal influence - Lazarsfeld y Katz, 1955) evidenciaba un "desbordamiento de fronteras" (Bauer, 1959). El reto de afrontar desde la investigación social los cambios de la época situaban de nuevoa los usos de la teoría bajo la posibilidad de unafragmentación en grandes sistemas, situación que el propio Merton había diagnosticado como "balcanización de la sociología" (Merton, 1949). Surgen en esta época diferentes propuestas para explicar y dar a comprender la nueva situación de los procesos comunicativos:el estructuralismo, desde sus raíces semióticas (Fabbri, 1973), los Cultural Studies (Hoggart, 1957) bajo la renovación de la izquierda (New Left), los Technology Studies a partir de las propuestas deMarshall McLuhan (1962 y 1964), o el resurgir de las 
sociologías interpretativas como propuesta de mirada hacia lo cotidiano(Goffmann, 1959).

Frente a esta diversificación de los usos de la teoría que ponía en riesgo la unidad ganada, el modelo estructural-fucnionalista propondría un nuevo espaciode sistematización conceptual en torno a la idea de paradigma, a modo de agrupación de teorías de rango medio consistentes entre sí. Se trata de una propuesta de sistematización que, aun resultando más laxa, ofrece una alta eficacia en su capacidad de codificación de la teoría ("codification of sociological theory" - Merton, 1968: 69-72) a partir de la integración de teorías parciales solidarias. En el ámbito de la comunicación, el clásico The Effects of Mass Communication de Joseph T. Klapper (Klapper, 1960), resulta unapieza fundamental para comprender esta reorganización de la disciplina bajo el modelo de paradigma de investigación, incluyendo modelos teóricos afines para la investigación de efectos mediáticos: the two-step flow of communication, Personal Influence, Opinion leaders mediate decisively between mass communicators and audiences, Theory of the Minimal Effects y Short term 'attitude change'. Con ello, se consolida el modelo de un uso de la teoría como teorías de rango intermedio de carácter instrumental, explicativo y predictivo, agrupadas conforme a un paradigma de investigación con voluntad de hegemonía, a modo de paradigma dominante de investigación de la comunicación.

\section{LOS USOS DE LA TEORÍA EN TIEMPOS DE GLOBALIZACIÓN: EL} PARADIGMA DOMINANTE ANTE EL SIGLO XXI

Tal y como planteábamos en las líneas precedentes, al igual que para analizarel desarrollo inicial y consolidación del paradigma dominante como investigación administrada y síntesis teórica a través de teorías de rango intermedio debíamos acudir a su contexto tecnológico, político y económico más inmediato, la actual forma que adopta dicho paradigma debe ser también puesta en relación con la realidad que contribuye radicalmente a su definición. Es por ello por lo que, desde finales de los años ochenta e iniciado ya el siglo xxI, es preciso hablar de los usos de la teoría en tiempos de globalización; una globalización que abarca el ámbito cultural, político-económico, tecnológico e incluso institucional.

Nos situamos ante un mundo interconectado globalmente en el que, tras la caída del Telón de Acero y el derrumbe de la Unión Soviética, el modelo de cultura mediática propiamente estadounidense ha consolidado su pretendida (desde los tiempos de la Guerra Fría) hegemonía, mundializándose formatos audiovisuales, contenidos y flujos comunicativos. Fruto de ese 
proceso, se establece un modelo cultural único, bajo la forma de diversas industrias culturales transnacionales que, a pesar de su pluralización ( $\mathrm{y}$, con ello, la pérdida del componente crítico del concepto - Carrasco-Campos y Saperas, 2011), proponen, tal y como pronosticaron Adorno y Horkheimer (1988), una aparente diversidad (definida actualmente como estilos de vida) que encubre la homogeneidad subyacente de la masscult norteamericana (MacDonald, 1969), en tanto que sociedad de consumo.

En este proceso de globalización, la innovación tecnológica, especialmente a través de la World Wide Web y su propuesta de públicos activos interconectados (idealmente capaces de participación horizontal en el sistema de flujos comunicativos), lejos de romper con ese esquema de homogeneización cultural habría conseguido integrar a los propios usuarios como copartícipes de las rutinas de producción, distribución, uso y consumo de productos y contenidos mediáticos y culturales. La paulatina extensión de una red cada vez más tupida en la que el ideal de intercomunicación mundial (o públicos y audiencias interconectados mundialmente) sería posible sólo en virtud de un mismo proceso global de socialización mediática, mediante la homogeneización y generalización un mismo esquema cultural compartido (en forma de sociedad global de consumo mediático conforme a una misma lógica de pseudo-cultura - Adorno y Horkheimer, 1979), bajo cuyo paraguas cabrían diferentes concreciones culturales desarraigadas de lo local,en constante proceso de descentralización y desterritorialización. Adicionalmente, pero de manera determinante, esta globalización de modelos culturales y mediáticos es posible también gracias a la mundialización de las estructuras económicas bajo la forma de un capitalismo neoliberal de carácter informacional (Castells, 1999), para el que la información, el conocimiento y la son mercancías intangibles para el intercambio en un mercado global.

En este contexto de globalización cultural, tecnológico-mediática y económico-política el paradigma dominante de investigación ha sufrido un tercer proceso de necesaria renovación, fruto de la aparición de nuevos fenómenos mediáticos, cambios en la propia estructura de medios y en las políticas e intereses económicos que los regulan, y cambios en las audiencias y públicos que los articulan. Así, desde los años ochenta han aparecido nuevas propuestas de sistematización teórica, no obstante basadas en el propio modelo inicial construcción teórica a partir de teorías de rango medio. De estos esfuerzos, debemos reseñar la importancia y sintomática relevancia del caso de la revista Journal of Communication, publicación vinculada a la International Communication Association (ICA), un caso escogido por 
la especial tarea de sistematización teórica del campo que propuso a través de dos números monográficos.

En primer lugar, destacamos la importancia y repercusión del especial Ferment in the Field de 1983, a cargo de George Gebner (Gerbner \& Siefert, 1983), número que sirvió para, en un contexto de incipiente globalización que amenazaban con una posible nueva balcanización de la teoría, procurar marcos internacionales comunes para los diferentes investigadores y centros de investigación. Según reconoce Robert T. Craig, miembro destacado de la ICA, con este monográfico se encauzaban las diferentes tradiciones investigadoras que, en un momento propicio para posibles rupturas, amenazaban la hegemonía del paradigma dominante y, por tanto, la homogeneidad del campo (Craig, 1999). La hoja de ruta para evitar posibles nuevos"rebasamientos de fronteras" pasaba, como años atrás, por fortalecer las posibilidades de las teorías intermedias y parciales como modelos de integración. En 1993, diez años más tarde, de nuevo el Journal of Communication pretendería intentar ordenar y sistematizar el ámbito con el especial The disciplinary Status of Communication Research, editado por Mark R. Levy y Michael Gurevitch (Levy y Gurevitch, 1993). Como en el caso anterior, el objetivo fundamental pretendía destacar los rasgos distintivos para la homogeneidad investigadora en el ámbito de la comunicación. Sin embargo, la estrategia en este caso se demostraba como una vía más débil, pero igualmente efectiva, de aceptación de una pluralidad teórica (siempre dentro de los márgenes de los modelos de rango intermedio), aunque bajo el dominio del empirismo.

En alianza tácita con esa aceptación de pluralismo teórico, dentro de los márgenes del empirismo y del carácter intermedio de las teorías, podemos encontrar también el artículo Communication Research Since Lazarsfeld de Elihu Katz (1987), en cuyas conclusiones se reconocen los primeros pasos hacia la institucionalización de la investigación como nueva forma de paradigma dominante. Asimismo,dos libros de referencia merecen también mención explícita puesto que con un marcado afán de sistematización, recogen los múltiples modelos teóricos aceptados como propios por la investigación de la comunicación. Nos referimos a los influyentes Milestones in mass communication research de Shearon Lowery y Melvin Lawrence De Fleur (Lowery y De Fleur, 1995) y Mass Communication Theory de Denis McQuail (McQuail: 2010), textos que cumplirán la función de lugar común, a escala internacional, para la docencia e investigación de teorías de la comunicación.

En líneas generales, estos nuevos esfuerzos para la sistematización de los usos de la teoría persisten en el modelo de investigador meramente instrumental que consolida de manera definitiva el proyecto inicial de renuncia al 
valorde la capacidad de generalización de la teoría, en favor de teorías de rango medio y sumisión al objeto de estudio mediante aproximaciones metodológicas estandarizadas. La tendente pérdida del valor de la teoría como fin en sí mismo (bajo intereses comprensivos o emancipatorios) se advertiría también en la primacía de los aspectos metodológicos en la investigación comunicativa actual, así como en la denominación de disciplinas por remisión pura a ámbitos y objetos de estudio particulares: Media Studies, Communication Studies, Technological Studies, Communication System Studies, Gender Studies, Political Communication Studies, Journalism Studies, o Public Relations Studies.

De nuevo, tal y como proponíamos páginas atrás, esta realidad debe ser contextualizada en el marco de globalización previamente descrito y analizado. Se trata de un marco que será determinante a la hora de diseñar los procesos investigadores y de definir, conforme a ellos, los usos de la teoría. Tal y como reconocen W. Lance Bennet y Robert M. Entman, en su presentación como editores de la serie Communication, Society and Politics de la Cambridge University Press (disponible en Hallin y Mancini, 2012), los cambios sociales (en especial los tecnológicos) suponen retos investigadores de diferente naturalezaque demandan un punto de vista global, mediante la comparación de los sistemas mediáticos del mundo, para el que son necesarias nuevas perspectivas teóricas rigurosas con base empírica ("new, rigorous, empirically grounded theoretical perspectives"). En esa necesidad de búsqueda de perspectivas teóricas subordinadas a la investigación empírica que hagan posible estudios comparados se vislumbra el actual modelo dominante de canon único y hegemónico de investigación,susceptible de generar resultados válidos para análisis comparados de tipo cuantitativo, y de avance por replicación. Fuera de esos márgenes, en el actual contexto global, parecenreducidos los espacios académicos para cualquier uso de la teoría diferente a este modelo investigador.

\section{HACIA LA INSTITUCIONALIZACIÓN Y ESTANDARIzACIÓN DEL} PARADIGMA DOMINANTE

Hasta el momento hemos analizado las diferentes características y factores de orden contextual que condicionan de manera radical las actuales posibilidades de usos de la teoría en los procesos de investigación de la comunicación. No obstante, a modo de reflexión final, es preciso también aludir a un factor añadido (mencionado tan solo implícitamente) que, en alianza con los procesos de globalización cultural, neoliberal y de innovación tecnológica, 
propiciaría no solo la subsistencia y adaptación de las formas originales del paradigma dominante, sino además su consolidación y promesa de vigencia futura en el contexto internacional. Nos referiremos en este punto a la institucionalización académica del paradigma dominante de investigación de la comunicación. Tal y como señalábamos anteriormente, pero ahora de manera mucho más explícita, es necesario destacar cómo en este continuo proceso constante de sistematización y redefinición de los usos de la teoría las diferentes instituciones académicas habrían jugado un papel determinante, no sólo como difusoras de las propuestas, sino como agentes activos y espacios en los que llevar a cabo ese proceso.

Por una parte, la producción editorial académica ha acaparado un papel fundamental a través de los journals internacionales de referencia y de textbooks de editoriales universitarias del ámbito anglosajón. Esta función del mundo editorial académico no debe contemplarse como un espacio inocuo para la difusión de la producción científica, sino como todo un esquema regulativo acerca de lo que es posible o no publicarse. La propia estructura recomendada para este tipo de artículos marca de por sí una pauta determinada de investigación, orientada hacia la producción empírica y hacia la síntesis teórica a modo de benchmarking o state of the art, como formas instrumentales de marcos teóricos para la investigación aplicada. Así incluso llegaría a suceder con la propia investigación teórica, para la cual T. Hanitzsch (2013) propone un checklist para la autoevaluación de artículos para la revista Communication Theory (vinculada a la ICA), mediante la cual no sólo se establece implícitamente un esuqema de investigación teórica, sino también un modo concreto de hacer teoría según la cual se fomente esa perspectiva instrumental ("Is the need for a theory intervention justified?") bajo la subordinación a los objetos de estudio ("Does the article address a communication problem?") en un proceso de construcción teórica lineal y por acumulación ("Does the literature review identify meaningful points of departure?", "Does the article engage the relevant communication literatures?")

Debemos también destacar la función rectora de las asociaciones internacionales de profesionales de investigación en comunicacióncomo agentes de institucionalización y estandarización del paradigma dominante de investigación comunicativa. Este tipo de asociaciones, la mayoría de ellas con una voluntad integradora de toda la comunidad académica internacional, desarrolla diferentes funciones clave de cara a la homogeneidad de los procesos investigadores. Por una parte, marca una fuerte pauta en los procesos formales e informales de redes y rutinas de trabajo investigador, por cuanto se las supone un espacio de diálogo y trabajo común en un contexto globalizado 
(aun estructurando a los diferentes investigadores por criterios de secciones temáticas conforme a ámbitos y objetos de estudio). Por otra parte, actúan como legítimos y reconocibles agentes institucionales en el intercambio y difusión del conocimiento, no sólo mediante los papers publicados en sus journals internacionales, sino también a través de las International Conferences y sus respectivos proceedings. Adicionalmente, en tanto que interlocutores científicos válidos, las asociaciones se establecen como espacios de construcción científica desde la autonomía que les confiere el mismo hecho de constituirse como agrupaciones profesionales sectoriales. De tal modo, la legitimidad de su proyección social en un contexto de mundialización la encuentran en su propia naturaleza corporativa, sin tener que recurrir a vías de cooperación (bajo la forma de Ethic of Cooperation) con otro tipo de instituciones extra-académicas.

Asimismo, como agentes de los procesos de estandarización internacional de los procesos de investigación, es preciso también subrayar el papel de otros organismos (políticos o académicos) de certificación de la carrera investigadora, aprobación de financiación de proyectos de investigación, rankings públicos de universidades y centros de investigación, estandarización internacional de índices de impacto de la difusión de la actividad investigadora, etc. Este tipo de instituciones y estándares no sólo determinan y profesionalizan el canon investigador hegemónico, sino también (y en estrecha alianza con este factor) el propio devenir de las carreras investigadoras, con sus hitos correspondientes, en un contexto de competencia laboral global y de difusión mundial de los conocimientos generados.

Estos componentes institucionales nos remiten no sólo hacia la preponderancia de un modelo de investigación dominante, al que se asocia un único uso instrumental, parcial e intermedio de la teoría, sino también hacia cierta interiorización de dicho canon, a través de la adquisición de rutinas profesionales estandarizadas de carácter internacional, asimiladas desde el principio de su carrera por los investigadores. Esta interiorización imperativa nos devuelve la imagen de una hegemonía investigadora cada vez más intangible y soterrada, por cuanto su no aceptación conllevaría a medio plazo el silencio o no reconocimiento de determinadas trayectorias por parte del resto de la comunidad investigadora.

\section{SÍNTESIS FINAL}

A lo largo de las anteriores páginas hemos ofrecido una caracterización y contextualización de los modelos de construcción teóricas actuales en los 
estándares internacionales de investigación aplicada a la comunicación. Este análisis nos ha permitido valorar la radical importancia de los elementos de globalización tecnológica, cultural, política y económica, así como los procesos de institucionalización de la actividad investigadora, en la configuración de un canon investigador hegemónico en la Academia. Nos referíamos así hacia un modelo de usos de la teoría basado en la construcción y síntesis desde teorías de rango medio como conjunto de hipótesis de alta aplicabilidad y replicabilidad empírica, susceptibles de ofrecer resultados comparables a nivel transnacional mediante estándares investigadores comunes, y publicados a través de circuitos de difusión también comunes.

De manera resumida, podemos sintetizar este modelo dominante del uso de la teoría en la investigación de la comunicación actual a partir de las siguientes características:

- Predomino de modelos de investigación empíricos, con alta capacidad de replicación (generalmente mediante técnicas cuantitativas y/o experimentales) que favorecen la explicación, predicción y comparación de resultados.

- Predominio de modelos teóricos débiles, de tipo intermedio, instrumentales (centralidad del dato frente a la síntesis teórica) y con escasa capacidad de generalización.

- Predominio de modelos teóricos apolíticos, con escasa capacidad de proyección e intervención en lo social.

- Predominio de modelos teóricos integradores, capaces de dar cuenta de una gran variedad de fenómenos, objetos y efectos, y de determinar los límites del campo disciplinario.

- Pérdida de la autonomía de la teoría, en su dimensión estructural y organizativa del campo disciplinario.

Estas principales tendencias acentúan el ya advertido valor instrumental de la teoría, así como la implícita y paulatina imposición de un modelo único de ciencia social, con intereses especialmente explicativos, a través de la institucionalización, estandarización e internacionalización del canon investigador en un contexto global. Como corolario de este proceso es preciso advertir de nuevo la reducción del ejercicio teórico a tareas instrumentales, 
con el consecuente olvido o enmudecimiento de cualquier de alternativa de teorización que aspire a proponer intereses de investigación distintos a los explicativos (formulación de hipótesis, síntesis de estados de la cuestión, generalización de resultados). Nos encontramos, por tanto, ante el dominio internacional y legitimación institucional de procesos de investigación empíricos, descriptivos y predictivos, respecto a los que propuestas de teorización que aspiren a la comprensión o crítica de la realidad socialquedan relegados a segundos planos marginales, o acaso expulsados de los circuitos hegemónicos de actividad profesional investigadora.

\section{REFERENCIAS BIBLIOGRÁFICAS}

ADORNO, T.W. (2003). Consignas. Buenos Aires: Amorrortu. Primera edición en alemán de 1969.

ADORNO, T.W.; HORKHEIMER, M. (1979). Teoría de la pseudo-cultura. Sociológica. Madrid: Taurus, pp. 175-201. Edición original de 1959.

ADORNO, T.W.; HORKHEIMER, M. (1988). Dialéctica del iluminismo. Buenos Aires: Sudamérica. Edición original de 1944.

BAUER, A.R. (1959). Comments on "The State of Communication Research". Public Opinion Quarterly, 23 (1), pp. 14-17.

CARRASCO-CAMPOS, A.; SAPERAS, E. (2011). La institucionalización del concepto de industrias culturales en el proceso de debate sobre políticas culturales en la Unesco y el consejo de Europa (1970-1982). AdComunica, 2, pp. 143-159.

CASTELLS, M. (1999). La era de la información: economía, sociedad y cultura. vol. 1. Madrid: Siglo XXI.

CRAIG, R.T. (1999). Communication theory as a field. Communication Theory, 9 (2), pp.199-161.

FABBRI, P. (1973). Le comunicazioni di massa in Italia: sguardo semiotico e malocchio della Sociologia. Versus, 5.

GERBNER, G.; SIEFERT, M. (EDS). (1883). Ferment in the Field: Communication Scholars Adress Critical Issues and Research Tasks of the Discipline. Journal of Communication, 33 (3).

GITLIN, T. (1978). Media sociology: the dominant paradigm. Theory and Society, 6 (2), pp. 205-253.

GOFFMANN, E. (1959): The Presentation of Self in Everyday Life. Edinburgh: University of Edinburgh Social Sciences Research Centre Press.

HABERMAS, J. (1986). Ciencia y técnica como ideología. Madrid: Tecnos. Primera edición en alemán de 1968. 
HALLIN, D.; MANCINI, P. (EDS). (2012). Comparing Media Systems Beyond de Western World. Cambridge: Cambridge University Press.

HANITZSCH, T. (2013). Writing for Communication Theory. Communication Theory, 23 (1).

HOGGART, R. (1957). The uses of litteracy: aspects of working class life. Londres: Chatto \& Windus.

HORKHEIMER, M. (2003). Teoría tradicional y teoría crítica. Madrid: Amorrortu. Primera edición en alemán de 1937.

KATZ, E. (1987). Communication research since Lazarsfeld. Public Opinion Quarterly, 51, pp. S25-S45.

KEYNES, J.M. (1931). An open letter to President Roosevelt. The New York Times, 31 de diciembre.

KLAPPER, J.T. (1960). The Effects of mass communication. An analysis of research on the effectiveness and limitations of mass media in influencing the opinions, values, and behavior of their audiences. Nueva York: The Free Press.

LAZARSFELD, P.F. (1941). Remarks on Administrative Rearch and Critical Communications Research. Studies in Philosophy and Social Science, 9.

LAZARSFELD, P.F.; KATZ, E. (1955). Personal Influence, The Part Played by People in the Flow of Mass Communications. Glencoe (Illinois): The Free Press.

LEVY, M.R.; GUREVITCH, M. (EDs.) (1993). The disciplinary Status of Communication Research. Journal of Communication, 43 (3).

LOWERY, S.; De FLEUR, M.L. (1995). Milestones in mass communication research. White Plains (NY): Longman. Tercera edición.

MacDONALD, D. (1969). Masscult y Midcult. En VV.AA. La industria de la cultura. Madrid: Alberto Corazón. Edición original de 1960.

McLUHAN, M. (1962). The Gutenberg Galaxy: The Making of Typographic Man. London: Routledge \& Kegan Paul.

McLUHAN, M. (1964). Understanding Media: The Extensions of Man. New York: McGraw-Hill.

McQUAIL, D. (2010). Mass Communication Theory. Londres: Sage. Sexta Edición.

MERTON, R.K. (1949). On sociological theories of the middle range. En Social theory and social structure. New York: The Free Press, pp. 39-53.

MERTON, R.K. (1968). Social Theory and Social Structure. New York: The Free Press. Tercera edición ampliada. 